\title{
Fluorofenidone inhibits apoptosis of renal tubular epithelial cells in rats with renal interstitial fibrosis
}

\author{
Hui Yang (iD), Weiru Zhang (iD), Tingting Xie (iD), Xuan Wang (iD), and Wangbin Ning (ib)
}

Department of Rheumatology and Immunology, Xiangya Hospital, Central South University, Changsha, China

\begin{abstract}
This study aimed to investigate the mechanism of fluorofenidone (AKF-PD) in treating renal interstitial fibrosis in rats with unilateral urinary obstruction (UUO). Thirty-two male Sprague-Dawley rats were randomly divided into sham, UUO, UUO + enalapril, and UUO + AKF-PD groups. All rats, except sham, underwent left urethral obstruction surgery to establish the animal model. Rats were sacrificed 14 days after surgery, and serum was collected for renal function examination. Kidneys were collected to observe pathological changes. Immunohistochemistry was performed to assess collagen I (Col I) protein expression, and terminal deoxynucleotidyl transferase-mediated nick end-labeling staining to observe the apoptosis of renal tubular epithelial cells. The expression of Fas-associated death domain (FADD), apoptotic protease activating factor-1 (Apaf-1), and C/EBP homologous protein (CHOP) proteins was evaluated by immunohistochemistry and western blot analysis. AKF-PD showed no significant effect on renal function in UUO rats. The pathological changes were alleviated significantly after enalapril or AKF-PD treatment, but with no significant differences between the two groups. Col I protein was overexpressed in the UUO group, which was inhibited by both enalapril and AKF-PD. The number of apoptotic renal tubular epithelial cells was much higher in the UUO group, and AKF-PD significantly inhibited epithelial cells apoptosis. The expression of FADD, Apaf-1, and CHOP proteins was significantly upregulated in the UUO group and downregulated by enalapril and AKF-PD. In conclusion, AKF-PD improved renal interstitial fibrosis by inhibiting apoptosis of renal tubular epithelial cells in rats with UUO.
\end{abstract}

Key words: Fluorofenidone; Unilateral ureteral obstruction; Renal fibrosis; Apoptosis; Enalapril

\section{Introduction}

Renal interstitial fibrosis is the common pathway and pathological basis of chronic kidney diseases (CKDs) progressing to end-stage renal disease (ESRD). Its main features include tubular atrophy and deposition of a large amount of extracellular matrix (ECM) (1). The pathogenesis of renal interstitial fibrosis is complex. Studies have found that excessive apoptosis of renal tubular epithelial cells may be the critical cause of tubular atrophy, and persistent apoptosis limits the repair of renal function (2).

Fluorofenidone (1-(3-fluorophenyl)-5-methyl-2-(1H)pyridone, AKF-PD) is a novel compound drug designed at Central South University (Changsha, Hunan, China). It has proven to have therapeutic effects in treating multiple-organ fibrosis, such as kidney, liver, and lung (3), but the antifibrotic mechanism of AKF-PD is not very clear. Previous studies indicated that AKF-PD could inhibit inflammation $(4,5)$, oxidative stress (6), renal tubular epithelial cell transdifferentiation (7), and fibroblast activation (8). This study aimed to observe the effect of AKF-PD on renal tubular epithelial cell apoptosis and apoptotic signaling pathway in rats with unilateral urinary obstruction (UUO), so as to enhance the understanding of the pharmacological mechanism of AKF-PD.

\section{Material and Methods}

\section{Animals and model establishment}

A total of 32 male Sprague-Dawley rats, aged 6-8 weeks, weighing 180-220 g were purchased from SLAC Laboratory Animal Center (China). All rats were kept in the Experimental Animal Center of Central South University at a temperature of $25 \pm 2^{\circ} \mathrm{C}$, humidity of $55 \pm 2 \%$, and 12-h light/dark cycle. The Animal Center also provided Specific Pathogen Free (SPF) food and drinking water ad libitum. This study was approved by the Institutional Animal Care Committee of Xiangya School of Medicine, Central South University.

The rats were randomly divided into sham, UUO, UUO + enalapril, and UUO + AKF-PD groups after 1 week of adaptive feeding, and each group had eight rats. The UUO operation was performed as described in a previous study (9). The rats of the latter three groups underwent left 
ureteral ligation close to the pelvis under aseptic conditions, while in the sham group, the left ureter was isolated without ligation. The drugs were dissolved in $0.5 \%$ carboxymethyl cellulose sodium. Enalapril $\left(10 \mathrm{mg} \cdot \mathrm{kg}^{-1} \cdot \mathrm{day}^{-1}\right.$; Yangtze River Pharmaceutical Group Co., Ltd., China) and AKF-PD (500 $\mathrm{mg} \cdot \mathrm{kg}^{-1} \cdot \mathrm{day}^{-1}$; Xiangya School of Pharmaceutical Sciences) were given by gavage to rats on the day after surgery. The concentration of AKF-PD was based on our previous studies $(8,10)$. The sham and UUO groups received an equivalent amount of normal vehicle by the same method. All rats were sacrificed 14 days after surgery.

Blood samples were collected by heart punctures in rats under anesthesia. The sera were centrifuged at $956 \mathrm{~g}$ for $10 \mathrm{~min}$ at $4^{\circ} \mathrm{C}$. Serum creatinine (Scr) and blood urea nitrogen (BUN) were determined at the clinical laboratory of Xiangya Hospital according to manufacturer's instructions.

\section{Histopathological examination}

The obstructive kidney tissues were routinely fixed in formalin, embedded in paraffin, and sliced into 4- $\mu$ m-thick sections. Hematoxylin and eosin (HE) and Masson trichrome staining were used to estimate the degree of renal tubulointerstitial injury and collagen deposition. Ten fields of renal cortex per section were randomly chosen under $200 \times$ magnification by a digital camera (Leica, Germany) coupled to a light microscope (Leica DM 5000B, Germany). The images had a high-resolution, and Image-Pro Plus 6.0 software (Media Cybernetics, Inc., USA) was used for semiquantitative analysis. The assessment criteria of renal interstitial injury comprised eight indexes, including renal tubular epithelial cell vacuolar degeneration, tubular dilatation, tubular atrophy, red cell cast, protein cast, interstitial edema, interstitial fibrosis, and interstitial cellular infiltration. The injury index ranged from 0 to 3 , with the following definition: 0) normal; 1) mild change; 2 ) moderate change; and 3) severe change. The renal fibrosis index was evaluated using the scoring system as follows: 0 points: normal; 1 point: $<25 \%$ staining; 2 points: $25-50 \%$ staining; 3 points: $51-75 \%$ staining; and 4 points: $>75 \%$ staining (10).

\section{Immunohistochemistry}

Immunohistochemical staining was conducted for detecting the expression and distribution of Col I, FADD, Apaf-1, and CHOP in the obstructive renal tissue. Shortly after dewaxing and rehydration, the sections were soaked in $3 \%$ peroxide at room temperature for $20 \mathrm{~min}$. The gastric enzyme diluent was used for antigen retrieval in an incubator at $37^{\circ} \mathrm{C}$, followed by $3 \%$ bovine serum albumin to block the sections for $30 \mathrm{~min}$. Then, the sections were incubated with primary antibodies against Col I (1:200; Abcam, UK), FADD (1:200; Abcam), Apaf-1(1:200; Abcam), and $\mathrm{CHOP}$ (1:100; Abcam) in phosphate-buffered saline (PBS) overnight at $4^{\circ} \mathrm{C}$. The secondary antibody was added for $30 \mathrm{~min}$ at $37^{\circ} \mathrm{C}$ after the sections restored to room temperature naturally. PBS was used as a negative control instead of primary antibodies. Each section was observed under $200 \times$ magnification and 10 random fields were preserved. Five sections per group were randomly chosen for this study. The percentage of positive staining area in each field was calculated using Image-Pro Plus 6.0 software. The results were classified as follows: 0 point, normal; 1 point: $<25 \%$ staining; 2 points: $25-50 \%$ staining; 3 points: $51-75 \%$ staining; and 4 points: $>75 \%$ staining (11).

\section{TUNEL staining}

The apoptotic epithelial cells were detected using the TUNEL Reagent Kit (Roche Applied Science, USA). Briefly, paraffin sections were deparaffinized and rehydrated, and blocked in $3 \%$ peroxide at room temperature for $30 \mathrm{~min}$. Then, $20 \mu \mathrm{g} / \mathrm{mL}$ proteinase $\mathrm{K}$ was used to digest protein for $20 \mathrm{~min}$ at $37^{\circ} \mathrm{C}$. The sections were incubated with TUNEL reagents in the dark for $60 \mathrm{~min}$ at $37^{\circ} \mathrm{C}$, followed by transfer into converter-peroxidase in the dark for $30 \mathrm{~min}$. They were washed with PBS three times for 5 min after each step. Then, diaminobenzidine staining and hematoxylin counterstaining were performed. Finally, the sections were sealed using neutral gum. Ten fields were selected randomly from each renal tissue section under $400 \times$ magnification, and the number of apoptotic epithelial cells in each field was counted. The average value was calculated as the level of apoptosis. Five slices were chosen from each group.

\section{Western blot analysis}

The renal tissues were lysed in sodium dodecyl sulfate (SDS) lysis buffer (Beyotime, China) for extracting total protein, and the concentration of protein was determined using the bicinchoninic acid protein assay kit (Thermo Fisher, USA). Then, $40 \mu \mathrm{g}$ total protein was loaded into each lane and separated by SDS-polyacrylamide electrophoresis. The proteins in the SDS gel were transferred onto a polyvinylidene difluoride membrane (Millipore, USA), and the nonspecific binding protein was blocked in Trisbuffered saline with Tween 20 (TBST) buffer containing 5\% skimmed milk for $1 \mathrm{~h}$ at $37^{\circ} \mathrm{C}$. The membrane was incubated using primary antibodies against glyceraldehyde-3phosphate dehydrogenase (GAPDH, 1:5000; Abcam), FADD (1:100; Abcam), Apaf-1 (1:2000; Abcam), and CHOP (1:1000; Abcam) overnight at $4^{\circ} \mathrm{C}$ on a shaking table. Sequentially, the membrane was immersed in goat anti-rat immunoglobulin G (lgG; 1:5000; ABclonal, USA) or goat anti-rabbit IgG (1:5000; ABclonal) antibody for $1 \mathrm{~h}$ at room temperature. The membrane was washed three times using TBST for 10 min after each step. The protein bands were visualized using an enhanced chemiluminescence system detection kit (ECL; Amersham, USA) in a bioimage system (Bio-Rad, USA). The grayscale value was calculated using Image-Pro Plus 6.0 software. Five samples were selected randomly for western blot analysis. 


\section{Statistical analysis}

All data are reported as means \pm SD, and SPSS 19.0 statistical analysis software (IBM, USA) was used for data analysis. The comparison between groups was performed by one-way analysis of variance. Newman-Keuls multiple comparison test was used as the statistical post-test. A $P$ value $<0.05$ was considered statistically significant.

\section{Results}

\section{AKF-PD had no effect on the renal function in UUO rats}

Two rats were excluded from the study due to model failure or ileus-related death during the experimental period. The number of rats in each group at the end of the experiment was seven in the sham and UUO groups and eight in the UUO + enalapril and UUO + AKF-PD groups. Our results showed that Scr and BUN levels were significantly increased in the three experimental groups compared to the sham group $(P<0.05)$. However, no significant difference was observed between the UUO + enalapril group and the UUO + AKF-PD group $(P>0.05)$ (Figure $1 \mathrm{~A}$ and $B$ ).

\section{AKF-PD alleviated renal pathological injury of obstructed kidney in UUO rats}

No significant pathological changes were observed in the sham group under the light microscope. Moreover, vacuolar degeneration of renal tubular epithelial cells, interstitial inflammatory cell infiltration, and a large amount of collagen deposition in interstitial space were also observed in the UUO group. Different degrees of tubular atrophy and dilatation were seen in the kidneys of rats in the UUO group. The lesions were alleviated distinctly after treatment with enalapril or AKF-PD (Figure 2A and B).

The renal interstitial injury index showed that the score of the UUO group increased markedly compared with the sham group $(P<0.05)$ and decreased to various degrees after treatment with enalapril or AKF-PD $(P<0.05)$. Although the index in the UUO + AKF-PD group was slightly lower than that in the UUO + enalapril group, no significant difference was observed between these two groups $(P>0.05)$ (Figure 2C). This study also analyzed the degree of collagen deposition using Masson staining. The renal interstitial fibrosis index was higher in the three experimental groups than in the sham group $(P<0.05)$, and enalapril or AKF-PD significantly reduced collagen deposition to different extents compared with the UUO group $(P<0.05)$. However, the effect in the two treatment groups showed no significant difference $(P>0.05)$ (Figure 2D)

\section{AKF-PD downregulated the expression of COL I in renal interstitium of UUO rats}

Figure $3 \mathrm{~A}$ shows that the brown-stained Col I was localized slightly around renal vessels but hardly appeared in the renal interstitial space in the sham group. However, a large amount of Col I was deposited in the renal interstitium of rats in the UUO group. Treatment with enalapril or AKF-PD led to a significant reduction in the interstitial Col I deposition. The semiquantitative results of Col I in immunohistochemical sections are shown in Figure 3B. The expression of Col I was markedly upregulated in the UUO group compared to the sham group $(P<0.05)$. Both enalapril and AKF-PD could significantly downregulate the expression of $\mathrm{Col} I$ in the UUO group $(P<0.05)$, but the efficacy of AKF-PD was stronger than that of enalapril $(P<0.05)$.

\section{AKF-PD reduced the number of apoptotic renal tubular cells in UUO rats}

The TUNEL staining sections revealed only a few apoptotic tubular epithelial cells in the sham group, while a large number of positive-stained epithelial cells were seen in the UUO group. These cells mainly existed in the atrophic and dilated tubules. The number of apoptotic cells decreased with the administration of enalapril or AKF-PD, and the therapeutic efficacy of AKF-PD seemed to be
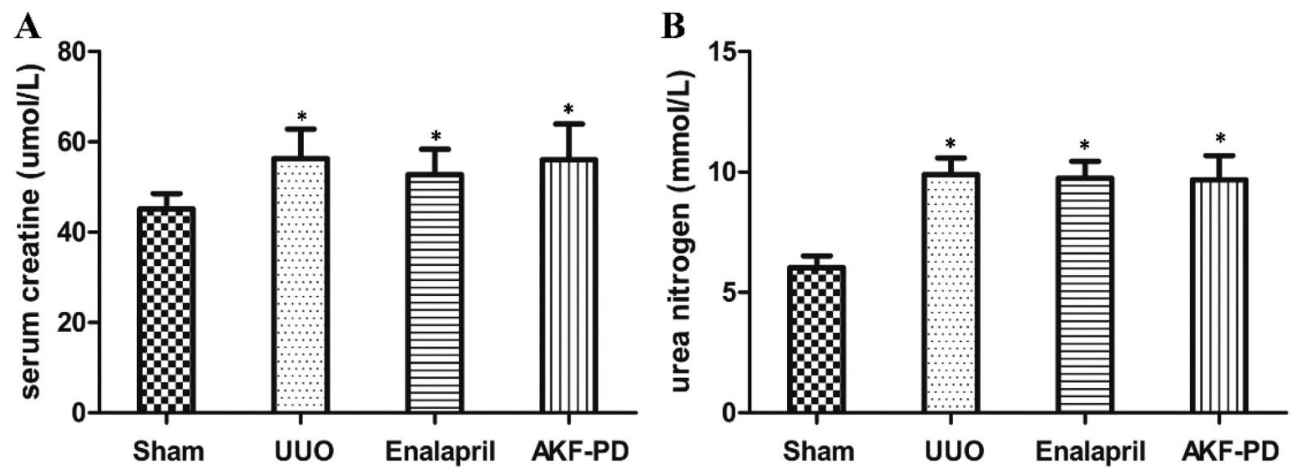

Figure 1. Fluorofenidone (AKF-PD) had no effect on renal function in the unilateral urinary obstruction (UUO) group. A, Semiquantitative analysis results of serum creatine and (B) urea nitrogen in the sham, UUO, UUO + enalapril, and UUO + AKF-PD groups. Data are reported as means $\pm \mathrm{SD}$. * $\mathrm{P}<0.05$ vs the sham group (ANOVA). 
A
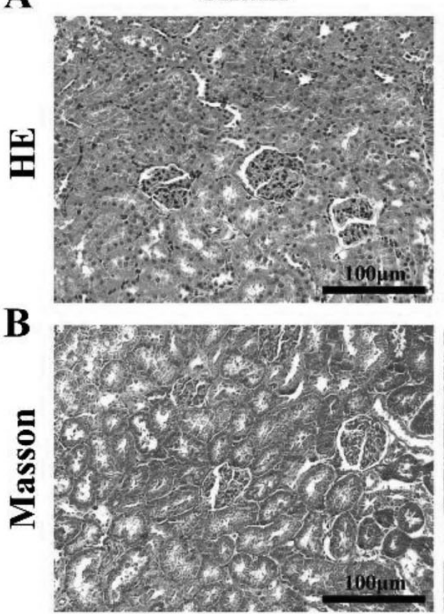

C

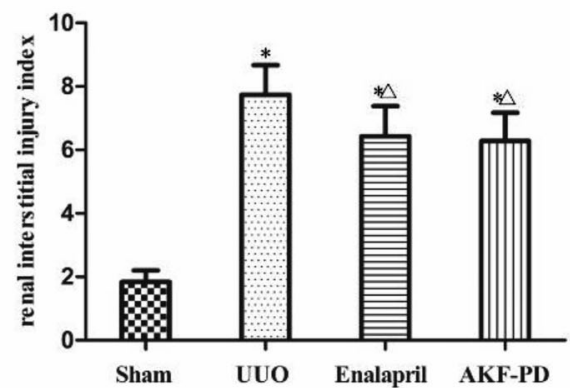

UUO
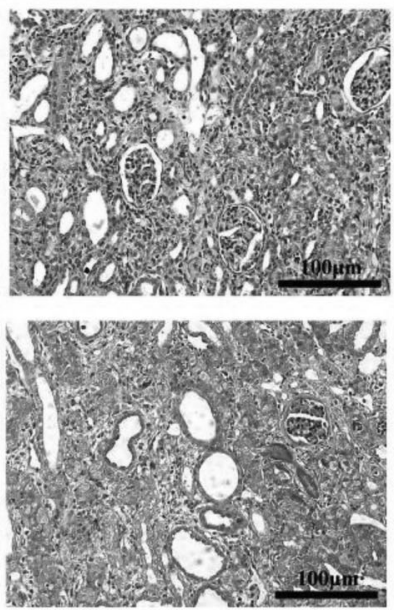

Enalapril
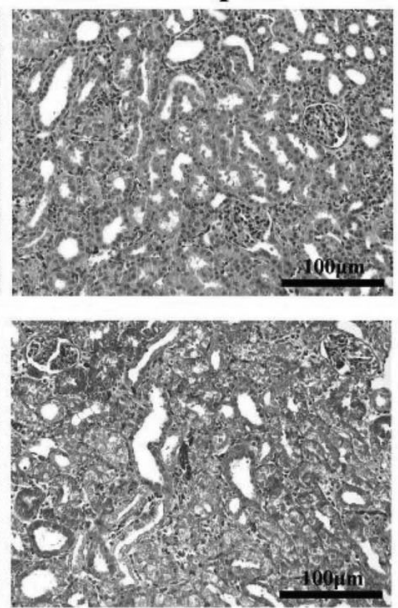

AKF-PD
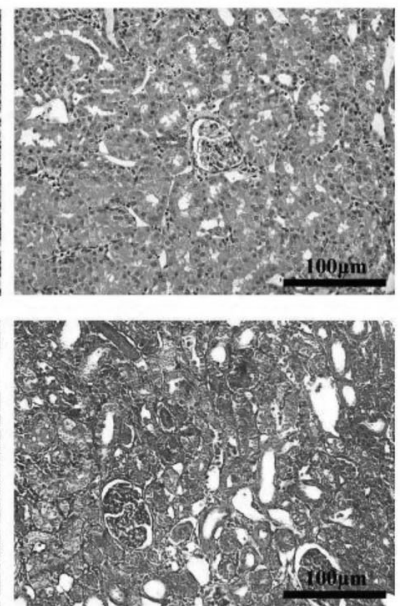

D

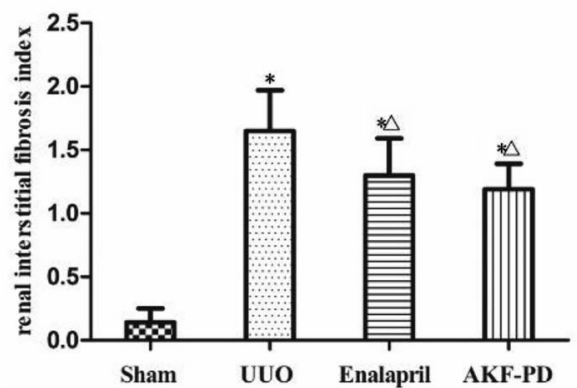

Figure 2. Fluorofenidone (AKF-PD) attenuated renal pathological changes in the unilateral urinary obstruction (UUO) group. A, HE staining and B, Masson staining results in the sham, UUO, UUO + enalapril, and UUO + AKF-PD groups $(200 \times$ magnification, bar: $100 \mu \mathrm{m})$. C, Semiquantitative analysis results of renal interstitial injury index, and D, renal interstitial fibrosis index. Data are reported as means \pm SD. ${ }^{*} \mathrm{P}<0.05$ vs the sham group. ${ }^{\triangle} \mathrm{P}<0.05$ vs the UUO group (ANOVA).

significant (Figure 4A). As shown in Figure 4B, the number of apoptotic cells increased in the UUO group compared to the sham group $(P<0.05)$, and enalapril and AKFPD suppressed tubular apoptosis to varying extents $(P<0.05)$. The reduction was more distinct in the UUO + AKF-PD group than in the UUO + enalapril group $(P<0.05)$.

\section{AKF-PD decreased the expression of FADD, Apaf-1, and CHOP proteins in kidney tissue of UUO rats}

Immunohistochemical staining showed only low expression of FADD, Apaf-1, and CHOP in the kidney tissue of rats in the sham group (Figure $5 \mathrm{~A}-\mathrm{C}$ ). After induction of UUO, the expression of these apoptotic proteins was upregulated significantly in the cytoplasm of atrophic or dilated tubular epithelial cells $(P<0.05)$. Compared with the UUO group, the number of positive-stained cells and protein expression levels of FADD, Apaf-1, and $\mathrm{CHOP}$ all decreased in the UUO + enalapril and UUO + AKF-PD group $(P<0.05)$. There was no significant difference between these two treatment groups $(P>$ 0.05) (Figure 5D-F).
To further confirm the effect of AKF-PD on apoptosisrelated proteins, the expression of FADD, Apaf-1, and $\mathrm{CHOP}$ in the renal tissues of the treatment and control groups was examined by western blot analysis. The developed bands and analysis results are displayed in Figure 6 . The aforementioned three proteins were lowly expressed in the sham group, and the expression increased in the rats of the UUO group $(P<0.05)$. After treatment with enalapril or AKF-PD, expression of FADD, Apaf-1, and CHOP distinctly declined in both groups compared with the UUO group $(P<0.05)$, but no statistical difference was found between the two treatment groups $(P>0.05)$.

\section{Discussion}

Renal fibrosis is the common pathway for the progression of various kidney diseases to ESRD, which is irreversible. Hence, inhibiting the process of renal fibrosis is the key to controlling the development of renal failure. Angiotensin-converting enzyme inhibitors and angiotensin receptor blockers are the traditional and 
A

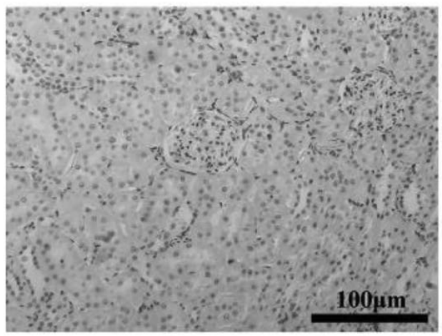

Enalapril

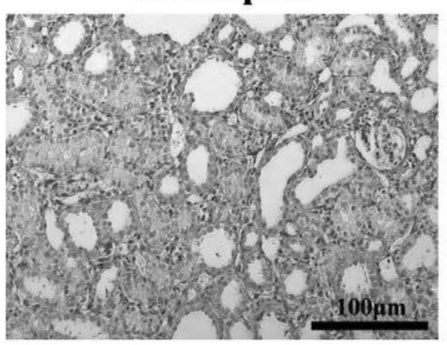

UUO

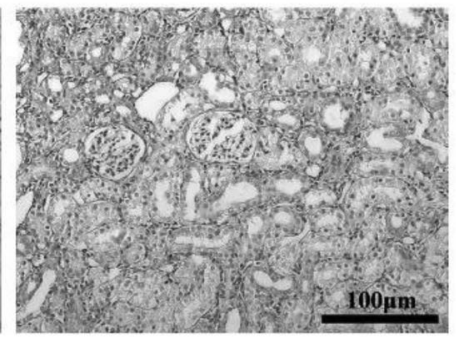

AKF-PD

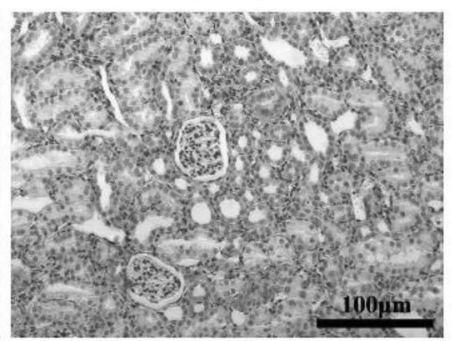

B

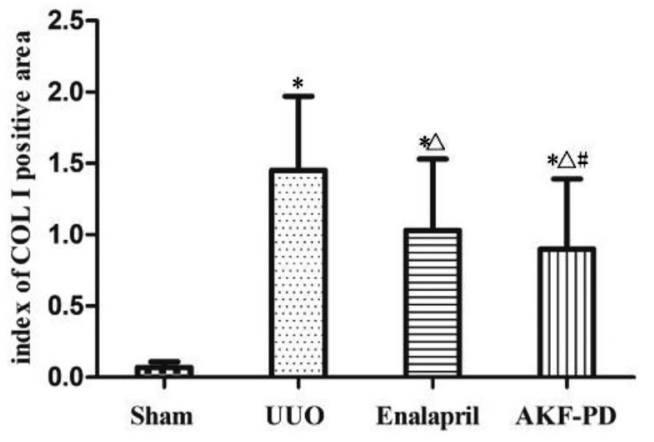

Figure 3. Fluorofenidone (AKF-PD) downregulated the expression of Col I in the renal interstitium of rats in the unilateral urinary obstruction (UUO) group. A, Immunohistochemistry results of Col I in the sham, UUO, UUO + enalapril, and UUO + AKF-PD groups $(200 \times$ magnification, bar: $100 \mu \mathrm{m})$. B. Semiquantitative analysis results of expression of Col I. Data are reported as means \pm SD. ${ }^{*} \mathrm{P}<0.05$ vs the sham group. ${ }^{\triangle} \mathrm{P}<0.05$ vs the UUO group. ${ }^{\#} \mathrm{P}<0.05$ vs the UUO + enalapril group.

$\mathbf{A}$

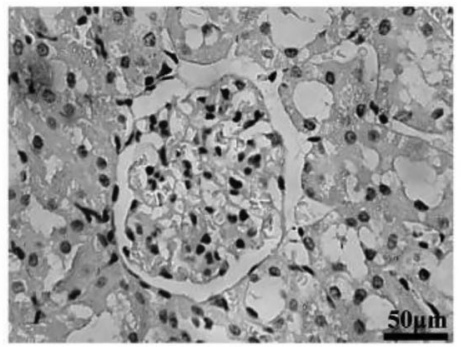

Enalapril

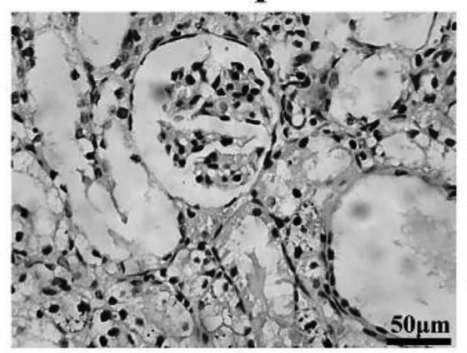

\section{UUO}

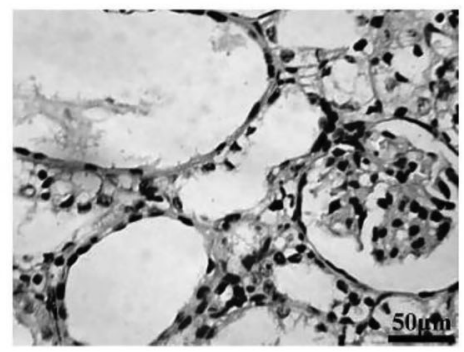

AKF-PD

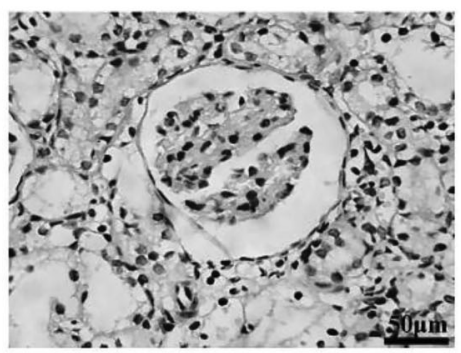

B

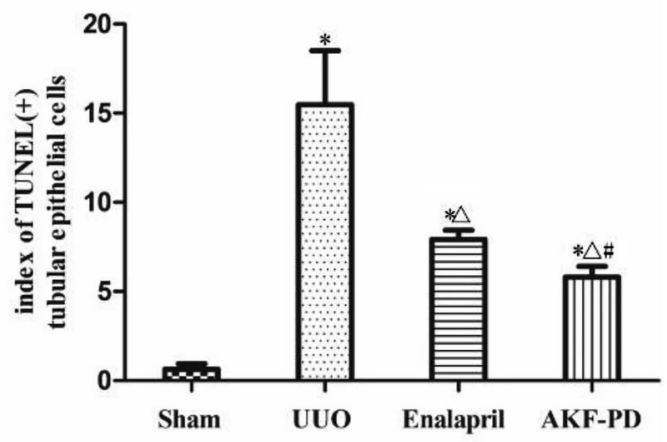

Figure 4. Fluorofenidone (AKF-PD) reduced the number of apoptotic renal tubular epithelial cells in the unilateral urinary obstruction (UUO) group. A, TUNEL staining results of kidney tissues in the sham, UUO, UUO + enalapril, and UUO + AKF-PD groups (400 $\times$ magnification, bar: $50 \mu \mathrm{m})$. B, Semiquantitative analysis results of the number of apoptotic tubular epithelial cells. Data are reported as means \pm SD. ${ }^{*} \mathrm{P}<0.05$ vs the sham group. ${ }^{\triangle} \mathrm{P}<0.05$ vs the UUO group. ${ }^{\#} \mathrm{P}<0.05$ vs the UUO + enalapril group (ANOVA).

widely clinically used renoprotective drugs, but they only delay the progression of renal failure other than blocking or reversing renal fibrosis (12). Patients with already developed ESRD can only be treated by renal replacement therapy, such as dialysis or kidney transplantation. Therefore, a new effective anti-fibrotic medicine 

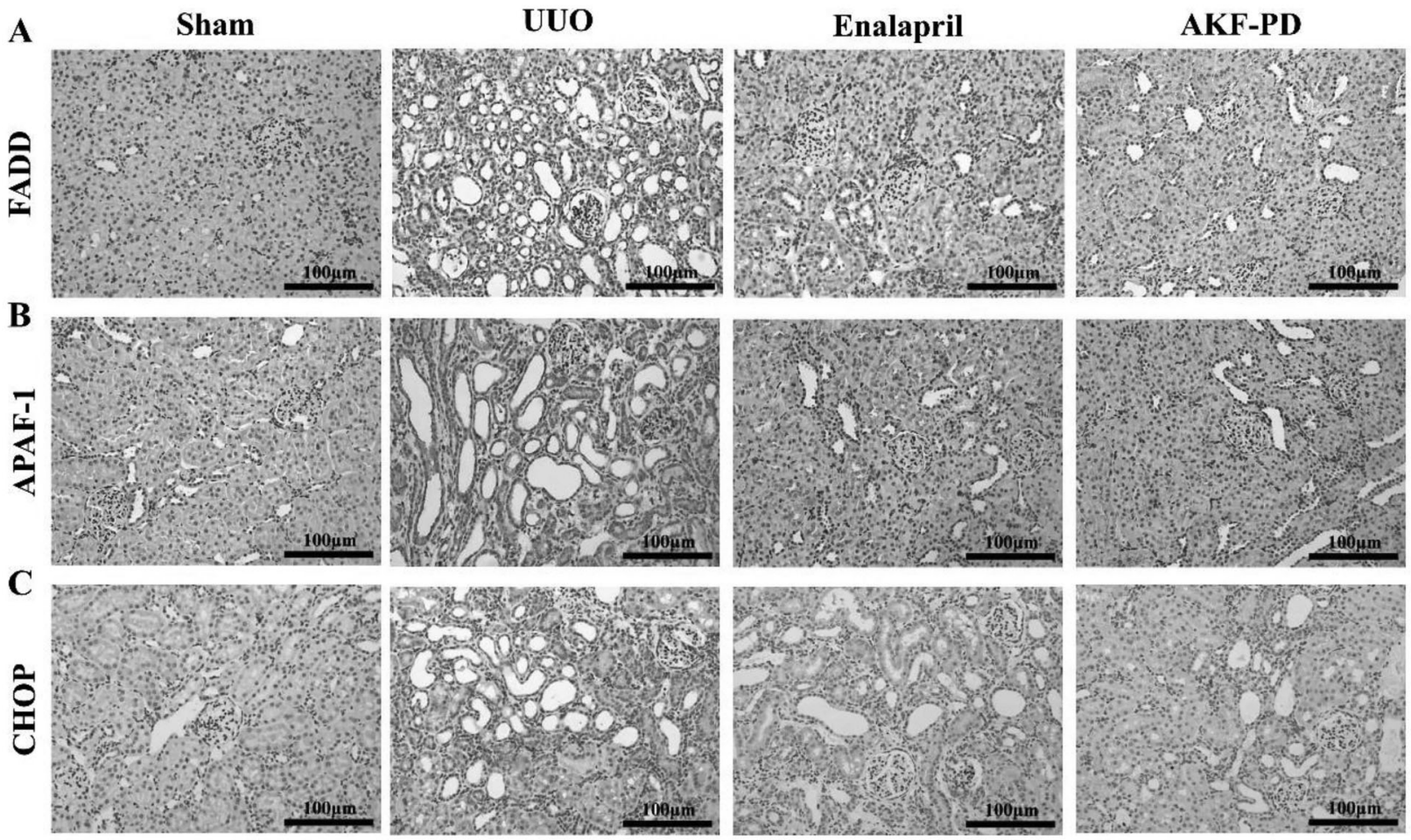

D

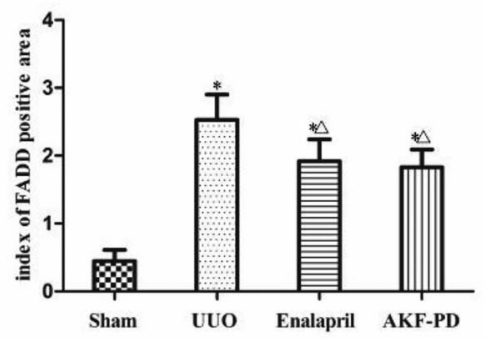

$\mathbf{E}$

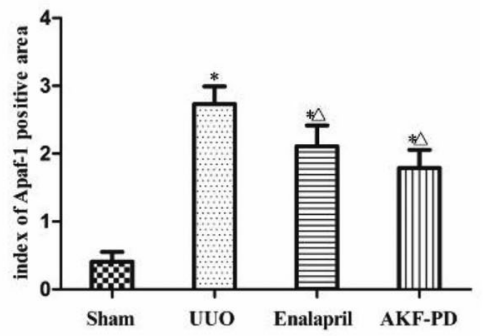

F

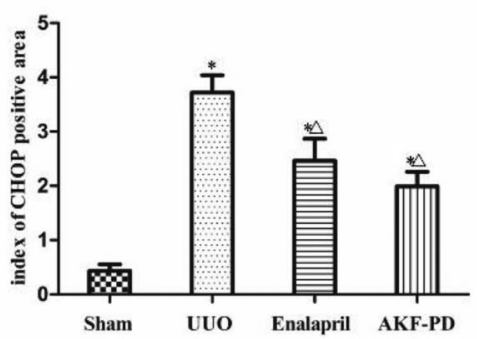

Figure 5. Fluorofenidone (AKF-PD) suppressed the protein expression of FADD, Apaf-1, and CHOP in the renal interstitium of rats in the unilateral urinary obstruction (UUO) group. Immunohistochemical results of FADD (A), Apaf-1 (B), and CHOP (C) in the sham, UUO, UUO + enalapril, and UUO + AKF-PD groups ( $200 \times$ magnification, bar: $100 \mu \mathrm{m})$. D, E, and F, Semiquantitative analysis results of the protein expression of FADD, Apaf-1, and CHOP. Data are reported as means $\pm S D$. ${ }^{*} P<0.05$ vs the sham group. ${ }^{\triangle} P<0.05$ vs the UUO group (ANOVA).

with multi-therapeutic pathways and targets is urgently needed.

AKF-PD is a pyridine compound having an anti-fibrotic effect similar to that of pirfenidone (13). Most of the preclinical studies of AKF-PD have been completed, and its application for phase I anti-hepatic fibrosis clinical trials is ongoing. Previous studies showed that AKF-PD had significant effects against renal interstitial fibrosis in many animal models, such as rats with UUO (10) and diabetic nephropathy (14). In this study, we proved that AKF-PD could ameliorate renal interstitial injury and fibrosis. These results indicated that AKF-PD had a renoprotective effect in rats with UUO. Col I is the main component of ECM, and the amount of collagen deposition in the renal interstitium represents the degree of fibrosis. In the present study, the expression of Col I protein was significantly downregulated by AKF-PD, further confirming that AKF-PD could relieve tubulointerstitial fibrosis.

The anti-fibrotic mechanism of AKF-PD is still largely unknown. Previous studies reported that it might occur by inhibiting the transdifferentiation of renal tubular epithelial cells and suppressing the proliferation and activation of fibroblasts in vivo $(7,8)$. AKF-PD could also alleviate renal fibrosis by downregulating the expression of fibrogenic cytokines, such as transforming growth factor$\beta$ and connective tissue growth factor, and blocking the 
A

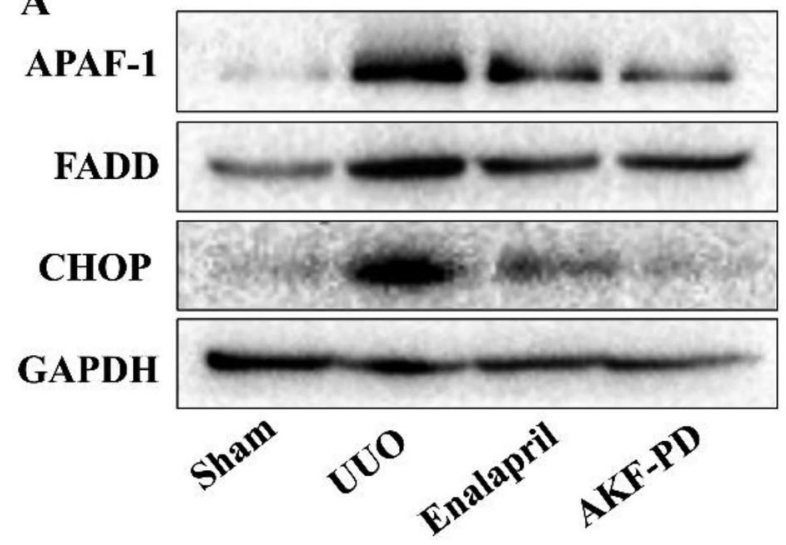

C

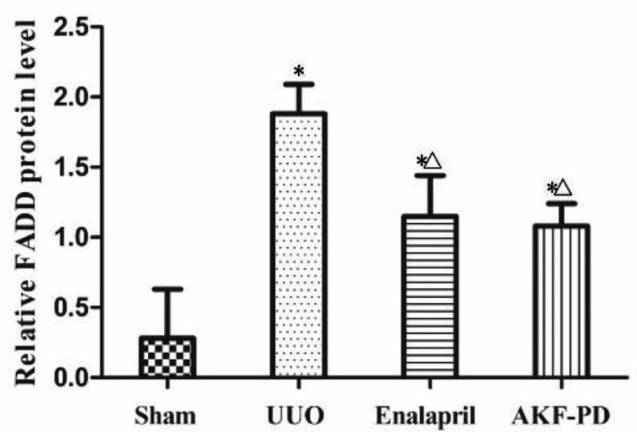

B

130KD

28KD

26KD

36KD

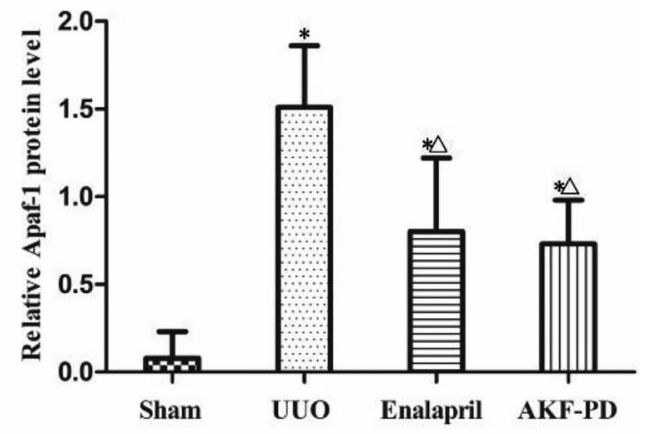

D

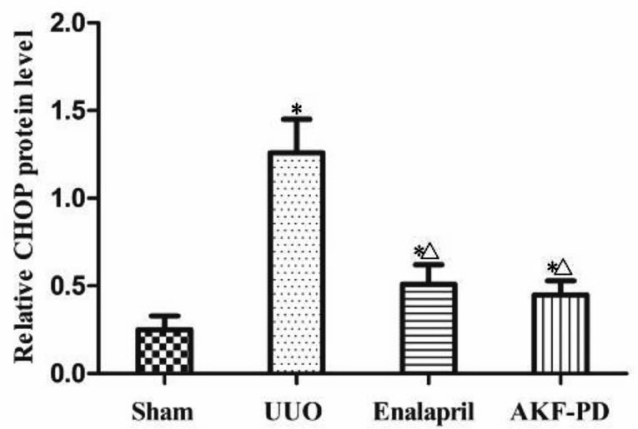

Figure 6. Fluorofenidone (AKF-PD) decreased the protein expression of FADD, Apaf-1, and CHOP in the kidney tissues of the unilateral urinary obstruction (UUO) group. A, Western blot analysis results of FADD, Apaf-1, and CHOP in the sham, UUO, UUO + enalapril, and UUO + AKF-PD groups. B, C, and D, Semiquantitative analysis results of the protein expression of Apaf-1, FADD, and CHOP, respectively. Data are reported as means $\pm S D$. ${ }^{*} \mathrm{P}<0.05$ vs the sham group. ${ }^{\triangle} \mathrm{P}<0.05$ vs the UUO group (ANOVA).

synthesis of Col I and Col III, promoting the degradation of ECM and inhibiting the oxidative stress and inflammatory response in vitro and in vivo (3). In vitro, AKF-PD could inhibit angiotensin II-induced apoptosis of renal tubular cells via blocking the Fas/FasL pathway (15) and attenuating apoptosis of NRK-52E cells induced by $\mathrm{H}_{2} \mathrm{O}_{2}$ (16).

Previous studies indicated that apoptosis of tubular epithelial cells is important in renal tubulointerstitial fibrosis. It was commonly believed that the main cause leading to tubular atrophy was excessive apoptosis of tubular epithelial cells after injury. The number of apoptotic epithelial cells was found to be significantly higher in patients with CKD than in normal people, and the number of apoptotic cells in renal tissue was related to renal interstitial injury index, renal fibrosis index, level of proteinuria, and deterioration of renal function (17). Other studies also reported that the increase in tubular epithelial cell apoptosis was apparently associated with interstitial fibrosis in UUO-operated rats, whereas inhibiting the apoptosis of tubular epithelial cells improved renal fibrosis (18). In this study, AKF-PD significantly relieved tubular epithelial cell apoptosis in rats with UUO, as detected by TUNEL staining, indicating that AKF-PD might improve renal interstitial fibrosis in rats with UUO by inhibiting the apoptosis of renal tubular epithelial cells.

Three pathways of apoptotic signal transduction, namely death receptor pathway, mitochondrial pathway, and endoplasmic reticulum pathway, have been reported to be involved in the occurrence and development of renal interstitial fibrosis.

The Fas/FasL pathway is the most classical death receptor pathway. During this process, activating FADD is the key step of this signaling pathway (19). The Fas/FasL pathway-related factors, such as Fas, FasL, and FADD, were upregulated in rats with UUO, while blocking the Fas pathway inhibited renal tubular epithelial cell apoptosis and renal interstitial fibrosis (20). Our research revealed that the expression of FADD protein in the renal tissue of rats in the UUO group was significantly reduced after AKF-PD treatment. It suggested that AKF-PD might inhibit renal tubular epithelial cell apoptosis by inhibiting the expression of FADD in the Fas/FasL pathway. 
The mitochondrial pathway, also known as the endogenous apoptotic pathway, also participates in the regulation of apoptosis. Apaf-1 activation is an important part of this process (21). Mitochondrial pathway-mediated apoptosis has been demonstrated in many animal models, including rats with UUO, and interrupting this pathway could significantly relieve renal interstitial fibrosis (22). The results of our study showed that the expression of Apaf-1 protein was greatly reduced in renal tissues of rats in the UUO group after AKF-PD treatment. It indicated that AKF$P D$ might also inhibit renal tubular epithelial cell apoptosis by inhibiting the expression of Apaf-1 in the mitochondrial pathway.

Endoplasmic reticulum pathway, also known as endoplasmic reticulum stress (ERS), has been proven to be involved in the pathogenesis of various fibrotic diseases, including renal fibrosis (23). The ERS-induced apoptosis is highly complex, and it is mainly mediated by $\mathrm{CHOP}$ (24). Studies showed that CHOP and other ERS signaling pathway-related molecules are overexpressed in rats in the UUO group, and inhibiting the expression of CHOP could improve renal interstitial fibrosis (25). Our study showed that the expression of CHOP protein was greatly reduced in renal tissues of rats in the UUO group after AKF-PD treatment. This indicated that AKF-PD might inhibit renal tubular epithelial cell apoptosis by inhibiting the expression of $\mathrm{CHOP}$ in the endoplasmic reticulum pathway.

Enalapril is a widely used angiotensin-converting enzyme inhibitor in clinics. It has remarkable renoprotective and antifibrotic effects on UUO rats (9). Thus, we

\section{References}

1. Boor P, Ostendorf T, Floege J. Renal fibrosis: novel insights into mechanisms and therapeutic targets. Nat Rev Nephrol 2010; 6: 643-656, doi: 10.1038/nrneph.2010.120.

2. Grgic I, Campanholle G, Bijol V, Wang C, Sabbisetti VS, Ichimura $\mathrm{T}$, et al. Targeted proximal tubule injury triggers interstitial fibrosis and glomerulosclerosis. Kidney Int 2012; 82: 172-183, doi: 10.1038/ki.2012.20.

3. Ma H, Peng Z, Hu G, Tao L. Effect and mechanism of fluorofenidone on organ fibrosis [in Chinese]. Zhong Nan Da Xue Xue Bao Yi Xue Ban 2015; 40: 208-213, doi: 10.11817/ j.issn.1672-7347.2015.02.016.

4. Huang $L$, Zhang F, Tang $Y$, Qin J, Peng $Y, W u ~ L$, et al. Fluorofenidone attenuates inflammation by inhibiting the NFKB pathway. Am J Med Sci 2014; 348: 75-80, doi: 10.1097/ MAJ.0000000000000187.

5. Tang $\mathrm{Y}$, Li B, Wang N, Xie Y, Wang L, Yuan Q, et al. Fluorofenidone protects mice from lethal endotoxemia through the inhibition of TNF-alpha and IL-1beta release. Int Immunopharmacol 2010; 10: 580-583, doi: 10.1016/j. intimp.2010.02.005.

6. Qin J, Mei W J, Xie YY, Huang L, Yuan QJ, Hu GY, et al. Fluorofenidone attenuates oxidative stress and renal fibrosis in obstructive nephropathy via blocking NOX2 (gp91phox) chose enalapril as the control in our study. Our previous study showed that AKF-PD, compared to enalapril, had a lower interstitial injury score and a better inhibitory effect on renal fibrosis indices such as Col I protein level, Col III mRNA and protein levels, and TGF- $\beta 1$ mRNA and CTGF protein expression (10). The present study further demonstrated that AKF-PD significantly decreased Col I protein level compared to enalapril. Also, AKF-PD exhibited a more potent effect of anti-apoptosis in renal tubular epithelial cells compared to enalapril. Moreover, it has been reported that AKF-PD can inhibit renal fibrosis via multiple pathways and targets (3). These results suggest AKF-PD may have advantages over enalapril in improving renal fibrosis.

In summary, AKF-PD inhibited the apoptosis of renal tubular epithelial cells in rats in the UUO group. This effect might be mediated by interfering with the expression of FADD, Apaf-1, and CHOP, which are key molecules in the Fas, mitochondrial, and endoplasmic reticulum pathways, respectively. However, the specific targets of AKF-PD in these three pathways need further investigation. The findings of this study suggested that the inhibition of tubular epithelial cell apoptosis might be a promising strategy for treating renal interstitial fibrosis.

\section{Acknowledgments}

This work was supported by the National Natural Science Foundation of China (81402993) and Hunan Provincial Natural Science Foundation of China (2018 JJ2638). expression and inhibiting ERK/MAPK signaling pathway. Kidney Blood Press Res 2015; 40: 89-99, doi: 10.1159/ 000368485.

7. Yuan Q, Wang L, Zhang F, Wang R, Fu X, Peng Z, et al. Fluorofenidone suppresses epithelial-mesenchymal transition and the expression of connective tissue growth factor via inhibiting TGF-beta/Smads signaling in human proximal tubular epithelial cells. Pharmazie 2011; 66: 961-967, doi: 10.1691/ph.2011.1535.

8. Yuan $Q$, Wang R, Peng Y, Fu X, Wang W, Wang L, et al. Fluorofenidone attenuates tubulointerstitial fibrosis by inhibiting TGF-beta(1)-induced fibroblast activation. $A m \mathrm{~J}$ Nephrol 2011; 34: 181-194, doi: 10.1159/000329080.

9. Kaneto H, Morrissey J, McCracken R, Reyes A, Klahr S. Enalapril reduces collagen type IV synthesis and expansion of the interstitium in the obstructed rat kidney. Kidney Int 1994; 45: 1637-1647, doi: 10.1038/ki.1994.215.

10. Li BX, Tang YT, Wang W, Xie YY, Wang NS, Yuan QJ, et al. Fluorofenidone attenuates renal interstitial fibrosis in the rat model of obstructive nephropathy. Mol Cell Biochem 2011; 354: 263-273, doi: 10.1007/s11010-011-0826-1.

11. Zhang QF. Ulinastatin inhibits renal tubular epithelial apoptosis and interstitial fibrosis in rats with unilateral 
ureteral obstruction. Mol Med Rep 2017; 16: 8916-8922, doi: $10.3892 / \mathrm{mmr} .2017 .7692$.

12. Boor P, Sebekova K, Ostendorf T, Floege J. Treatment targets in renal fibrosis. Nephrol Dial Transplant 2007; 22: 3391-3407, doi: 10.1093/ndt/gfm393.

13. Chen J, Lu MM, Liu B, Chen Z, Li QB, Tao LJ, et al. Synthesis and structure-activity relationship of 5-substituent$2(1 \mathrm{H})$-pyridone derivatives as anti-fibrosis agents. Bioorg Med Chem Lett 2012; 22: 2300-2302, doi: 10.1016/j. bmcl.2012.01.073.

14. Wang LH, Liu JS, Ning WB, Yuan QJ, Zhang FF, Peng ZZ, et al. Fluorofenidone attenuates diabetic nephropathy and kidney fibrosis in db/db mice. Pharmacology 2011; 88: 8899, doi: 10.1159/000329419.

15. Ning WB, Hu GY, Peng ZZ, Wang L, Wang W, Chen JY, et al. Fluorofenidone inhibits Ang Il-induced apoptosis of renal tubular cells through blockage of the Fas/FasL pathway. Int Immunopharmacol 2011; 11: 1327-1332, doi: 10.1016/j.intimp.2011.04.016.

16. Mei W, Peng Z, Lu M, Liu C, Deng Z, Xiao Y, et al. Peroxiredoxin 1 inhibits the oxidative stress induced apoptosis in renal tubulointerstitial fibrosis. Nephrology (Carlton) 2015; 20: 832-842, doi: 10.1111/nep.12515.

17. Teteris SA, Menahem SA, Perry G, Maguire JA, Dowling JP, Langham RG, et al. Dysregulated growth factor gene expression is associated with tubulointerstitial apoptosis and renal dysfunction. Kidney Int 2007; 71: 1044-1053, doi: 10.1038/sj.ki.5002176.

18. Docherty NG, O'Sullivan OE, Healy DA, Fitzpatrick JM, Watson RW. Evidence that inhibition of tubular cell apoptosis protects against renal damage and development of fibrosis following ureteric obstruction. Am J Physiol Renal Physiol 2006; 290: F4-F13, doi: 10.1152/ajprenal.00045. 2005.

19. Lee EW, Seo J, Jeong M, Lee S, Song J. The roles of FADD in extrinsic apoptosis and necroptosis. $B M B$ Rep 2012; 45: 496-508, doi: 10.5483/BMBRep.2012.45.9. 186.

20. Hauser P, Oberbauer R. Tubular apoptosis in the pathophysiology of renal disease. Wien Klin Wochenschr 2002; 114: 671-677.

21. Shakeri R, Kheirollahi A, Davoodi J. Apaf-1: Regulation and function in cell death. Biochimie 2017; 135: 111-125, doi: 10.1016/j.biochi.2017.02.001.

22. Xu Y, Ruan S, Wu X, Chen H, Zheng K, Fu B. Autophagy and apoptosis in tubular cells following unilateral ureteral obstruction are associated with mitochondrial oxidative stress. Int J Mol Med 2013; 31: 628-636, doi: 10.3892/ ijmm.2013.1232.

23. Chiang CK, Hsu SP, Wu CT, Huang JW, Cheng HT, Chang $\mathrm{YW}$, et al. Endoplasmic reticulum stress implicated in the development of renal fibrosis. Mol Med 2011; 17: 12951305, doi: 10.2119/molmed.2011.00131.

24. Vassart G, Brabant G, Costagliola S, Danguy D, Gérard C, Libert $F$, et al. Molecular genetics of the thyrotropin receptor. Exp Clin Endocrinol 1992; 100: 9-11, doi: 10.1055/s-00291211165.

25. Liu SH, Yang CC, Chan DC, Wu CT, Chen LP, Huang JW, et al. Chemical chaperon 4-phenylbutyrate protects against the endoplasmic reticulum stress-mediated renal fibrosis in vivo and in vitro. Oncotarget 2016; 7: 22116-22127, doi: 10.18632 /oncotarget.7904. 